\title{
A Case of Successful Colonoscopic Treatment of Colonic Obstruction Caused by Phytobezoar
}

\author{
Sang Seok Yoon, Min Seong Kim, Dong Yoon Kang, Tack Su Yun, Jun Ho Jeon, Yong Kyu Lee, \\ Se Woong Choi, Chi Hoon Kim \\ Department of Internal Medicine, Good Gang-An Hospital, Busan, Korea
}

\begin{abstract}
A phytobezoar is the most common type of bezoar, which is a gastrointestinal mass composed of vegetable. A persimmon is a common cause of a phytobezoar. The majority of bezoars are found in the stomach, with the small intestine being the next most commonly involved site. The colon is a rare site for a bezoar. Recently, we experienced a colonic bezoar that caused colonic obstruction in a 66-year-old female patient who took persimmons regularly. The patient came to the hospital because of abdominal pain and distension. To differentiate a tumor or other problems that can cause intestinal obstruction, we performed an abdominal computed tomography scan and found an ovoid intraluminal mass with a mottled gas pattern in the distal descending colon. A large impacted bezoar was seen in the sigmoid colon, which was completely obstructed, and it was successfully removed by using colonoscopy.
\end{abstract}

\section{Keywords: Bezoars; Intestinal obstruction; Colonoscopy}

\section{INTRODUCTION}

Bezoars are masses formed by the condensation of debris or stomach content in the gastrointestinal tract, and depending on the content, there are phytobezoars that are composed of plant material fibers, trichobezoars that are composed of hair, lactobezoars that are formed in infants from constant intake of inspissated milk, pharmacobezoars that are formed by sustained release medications, etc. [1-3]. Trichobezoars are the most commonly found bezoars in the Western world, but in Korea, phytobezoars make up the majority, and a high number of phytobezoars especially are formed mainly from the intake of persimmons $[1,3]$. The complications caused by bezoars are mechanical irritation, gastrointestinal obstruction, and, in severe cases, peritonitis caused by gastric perforation [3]. Obstructions of the gastrointestinal tract caused

Received: March 20, 2011 Accepted: June 30, 2011

Correspondence to: Sang Seok Yoon, M.D.

Department of Internal Medicine, Good Gang-An Hospital,

40-1 Namcheon-dong, Suyeong-gu, Busan 613-815, Korea

Tel: +82-51-610-9615, Fax: +82-51-621-1500

E-mail: orga001@gmail.com

(C) 2011 The Korean Society of Coloproctology

This is an open-access article distributed under the terms of the Creative Commons Attribution NonCommercial License (http://creativecommons.org/licenses/by-nc/3.0) which permits unrestricted noncommercial use, distribution, and reproduction in any medium, provided the original work is properly cited. by bezoars are about $0.4-4 \%$ of the total obstructions and mainly occur in the stomach or small intestine [4]. The occurrence of colonic obstructions caused by bezoars is considered pretty rare [4]. The authors of this study diagnosed a colonic obstruction caused by a phytobezoar and removed that obstruction by using colonoscopy. In this paper they report their experience with that case, along with a literature review.

\section{CASE REPORT}

A 66-year-old female patient was admitted for abdominal discomfort that had started a month before. She had been treated usually for constipation, and she complained of recent reduced stool bulk and residual stool. After taking lactulose, her abdominal pain and abdominal distension became worse. She stated that, up until her admittance, her meals had been usual, and that she often ate persimmons. Past illnesses included her receiving a laparoscopic sterilization procedure when she was 32 years old, and treatment for hypertension during the last 5 years. She had been diagnosed with a cerebral infarction 2 years prior and had been taking anticoagulants. Her blood pressure at admittance was $160 / 120 \mathrm{mmHg}$, her pulse 72 beats per minute, and her body temperature $36.8^{\circ} \mathrm{C}$. She had a protuberant abdomen, and although she had abdominal tenderness, there was no rebound tenderness, and she had increased bowel sounds. On rectal examination, no hemorrhoids or masses 
were palpable. In the peripheral blood test, hemoglobin was 13.4 $\mathrm{g} / \mathrm{dL}$, white blood cells were $12,310 / \mathrm{mm}^{3}$ (neutrophils, $83 \%$ ), and hematocrits were $41.0 \%$. In the serum biochemistry test, AST was $40 \mathrm{U} / \mathrm{L}$, ALT was $30 \mathrm{U} / \mathrm{L}$, ALP was $580 \mathrm{U} / \mathrm{L}$, and r-GTP was 252 $\mathrm{U} / \mathrm{L}$; no abnormalities were found on other tests.

In the plain abdominal X-ray finding, the areas proximal to the small intestine and the colon were expanded, and there were multiple air-fluid levels (Fig. 1A, B). In the abdominal computed tomography, the areas proximal to the small intestine and the colon were distended, and in her rectal sigmoid colon, a spherically-shaped mass that was about $3.5 \mathrm{~cm}$ in size, with a mottled gas pattern and that had been calcified was observed. There were no thickened walls or masses that would be suspected of being cancerous tumors
(Fig. 2).

In the first phase, although an enema was attempted to emit stools through bowel emission, stool did not come out; because her symptoms continued, a colonoscopy was performed. The colonoscopy revealed a foreign mass surrounded with fibrous materials (Fig. $3 \mathrm{~A}$ ), and this foreign mass was blocking the sigmoid colon rectal junction region; because the upper part of this mass was not in the endoscope, it was not observable. The stools surrounding this foreign mass were washed away, and, by using forceps, a snare, and a tripod, we scraped the surface of this mass, after which we used a net to eliminate the foreign mass (Fig. 3B). The emitted foreign mass, a hard brown lump, was approximately $4 \times 5 \mathrm{~cm}$ and was identified as a bezoar surrounded by fibrous materials (Fig. 3C).
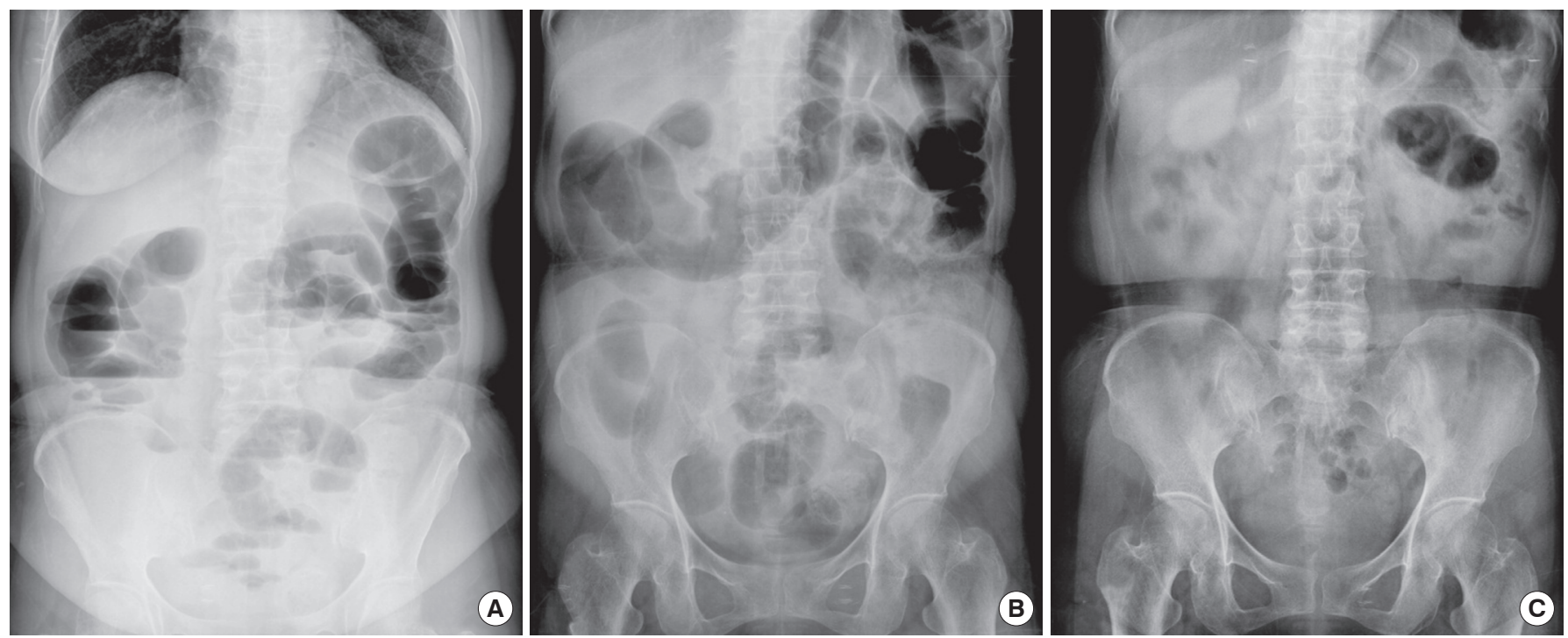

Fig. 1. (A) Erect simple abdomen showing dilated loops of the small and the large intestine swith multiple air-fluid levels. (B) Supine simple abdomen showing a dilated loop of the large intestine. (C) After removal of the bezoar, the ileus was improved.
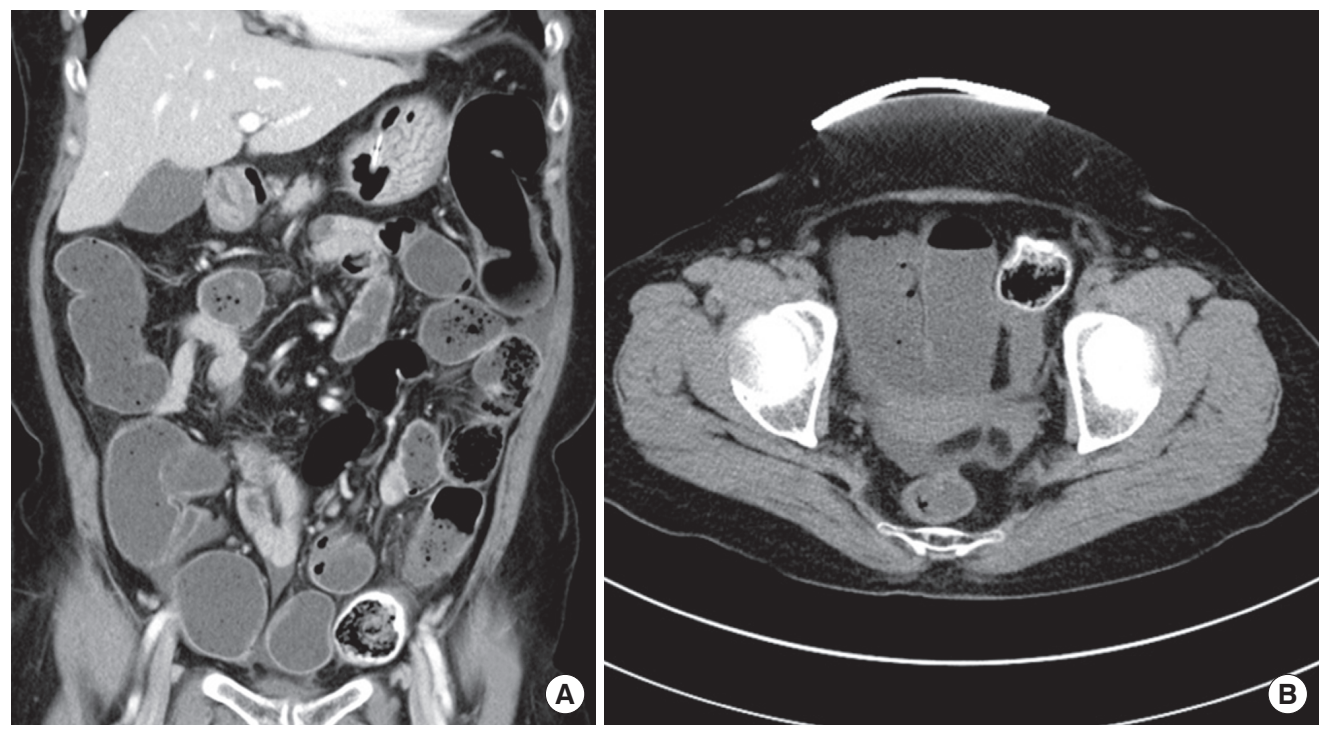

Fig. 2. (A) Coronary view showing distended large bowel loops up to the distal descending colon where a well-defined ovoid intraluminal mass with a mottled gas pattern is seen. (B) Transverse view showing an ovoid intraluminal mass in the distal colon with a distended large bowel loop. 

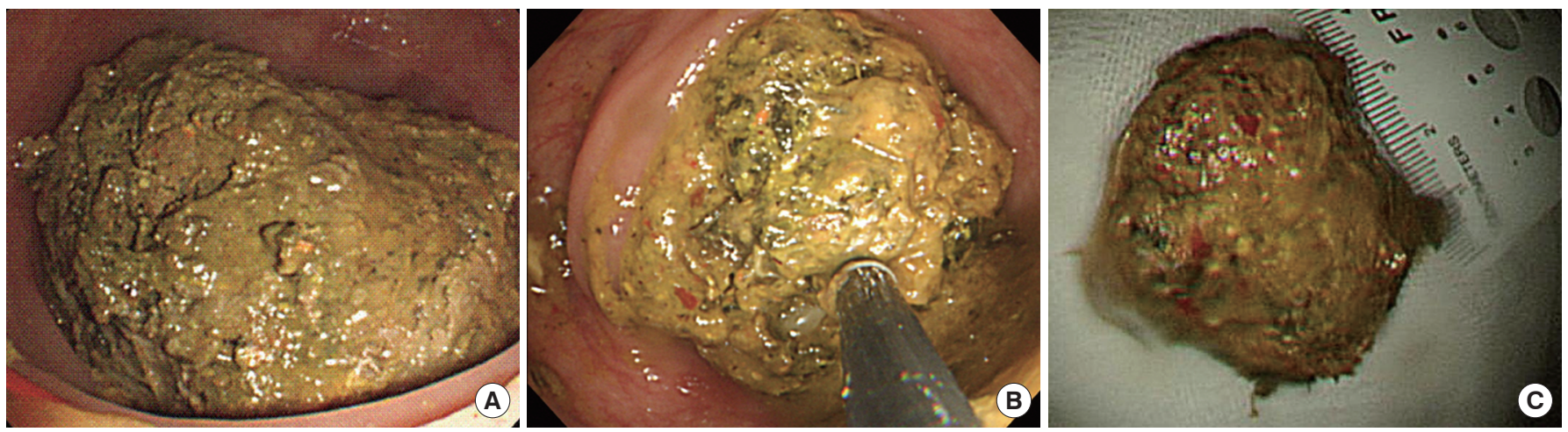

Fig. 3. (A) Impacted bezoar seen with complete obstruction is seenon colonoscopy. (B) The bezoar was caught with a tripod and removed from the large intestine. (C) A $4 \times 5 \mathrm{~cm}$ round-shaped bezoar was removed from the colon by using colonoscopy.

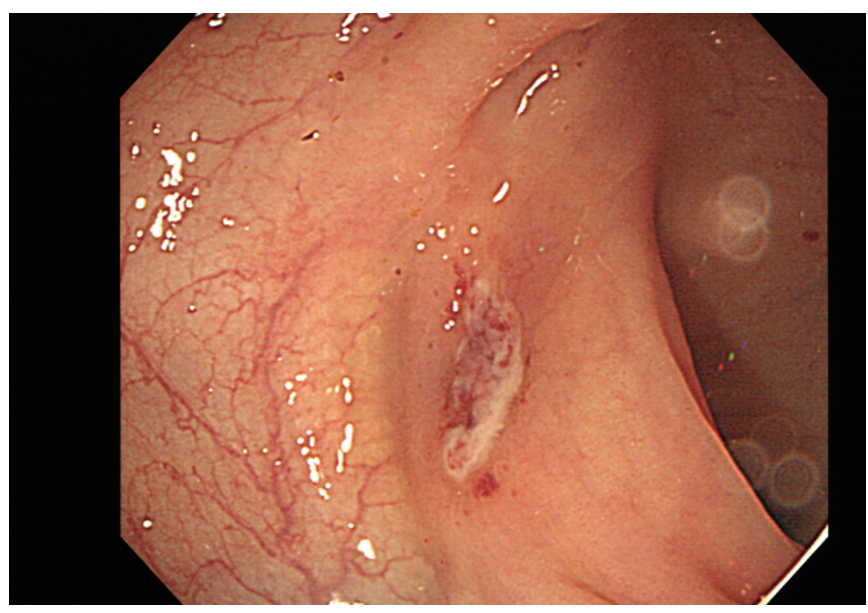

Fig. 4. Sigmoidoscopic finding. An ovoid-shaped ulcer is seen at the previous bezoar site.

After the removal of the foreign mass, abdominal pain and abdominal distension improved, and the next day, a plain abdominal $\mathrm{x}$ ray was taken and showed that the enlarged gastrointestinal tract had been significantly reduced (Fig. 1C). To ascertain the presence of bezoar remnants and other causes for obstruction such as malignant tumors, we performed a colonoscopy again; although no other potential causes for obstruction were observed, ulcers--likely caused by pressure-were present in the area where the bezoar had been (Fig. 4). One month later, sigmoidoscopy confirmed that the ulcers had been completely healed.

\section{DISCUSSION}

Bezoars are masses formed by the condensation of debris or stomach content in the gastrointestinal tract, and depending on the content, there are phytobezoars that are composed of plant material fibers, trichobezoars that are composed of hair, lactobezoars that are formed in infants from constant intake of inspissated milk, pharmacobezoars that are formed by sustained release medica- tions, etc. [1, 2]. In Korea, phytobezoars caused by persimmons are found most often, and the percentage of phytobezoars caused by persimmons ranges, depending on study results, from 30\% to $90 \%$ [1-3]. The mechanisms of phytobezoar formation are the shibuol, a tannin in persimmons, being exposed to gastric acids, masses being formed, and these becoming bezoars as they combine with fibrous materials $[5,6]$. These persimmon phytobezoars, compared to other causes, commonly result in complications; therefore, they are often removed with surgery $[1-4,6]$.

The occurrence of phytobezoars is often proportional to the age of the patient; according to a 1983 report by Debakey and Ochsner, phytobezoars occur more than $70 \%$ of the time in patients above the age of 30 [7]. In addition, phytobezoars can occur more often when there are antecedents such as gastrointestinal surgery, diabetic gastropathy, muscular atrophy, and cerebral infarction, or when medications that decrease gastrointestinal mobility are used [8-10]. Patients who have received a proximal subtotal gastrectomy or a laparoscopic posterior truncal vagotomy, because of their reduced gastrointestinal mobility, reduced gastric acid secretion, and loss of function of pylori, are more susceptible to bezoar formation whereas in patients who have undergone other types of gastrointestinal surgeries, when there is intestinal diameter stenosis due to intestinal adhesion or when the smooth flow of intestinal contents is disrupted, phytobezoars can occur easily [3, 8-11]. In cases of patients with past illnesses such as diabetic gastropathy or muscular atrophy, because of the reduced gastrointestinal mobility, the time to discharge intestinal contents is increased, which leads to increased occurrence of bezoars [3]. In our case, the bezoar in the female patient is thought to be attributable to her past illnesses, she had undergone a laparoscopy and had received treatment for a cerebral infarction, and to her consumption of persimmons while her gastrointestinal mobility was reduced due to her taking beta blockers that can cause a reduction in gastrointestinal mobility.

The most common complication of bezoars is gastrointestinal obstruction, and in some cases, mucosal damage caused by the irritation by bezoars can occur [3]. Among the causes for an obstruction in the gastrointestinal tract, bezoars account for about $0.4-4 \%$, 
and mostly obstructions occur in the stomach or the ileum in the small intestine [4]. Obstruction almost never occurs in the colon, but albeit rare, as shown in this case study, it can occur in a physiological stenosis region like the rectal sigmoid colon junction [4, 6-12]. Stomach pain is the most common clinical symptom for bezoars in the colon, and sometimes other symptoms are abdominal distension, vomiting, constipation, diarrhea, palpable lumps, loss of appetite, weight loss, etc. [4, 6-12]. For the diagnosis of bezoars in the colon, simple abdominal X-ray and abdominal computed tomography scans are performed [13-15]. In the abdominal computed tomography, $89 \%$ of bezoars appear as spherically-shaped masses containing air-fluid levels; because stools appear as long solid lumps with no membranes, they are easily distinguishable from bezoars [16]. In addition, because abdominal computed tomography can discriminate not only bezoars but also other potential causes of gastrointestinal tract obstructions such as gastrointestinal malignancies and because it has higher sensitivity and specificity in diagnosing gastrointestinal obstructions compared to other tests, this method should be considered first [13-16]. In this case, in order to determine the cause of bowel obstruction, we used abdominal computed tomography, and it resulted in the confirmation of a foreign mass suspected to be a bezoar in the sigmoid colon junction.

The methods for removing bezoars in the colon can differ according to the location of the closure caused by the bezoar, the size of the bezoar, and the presence of complications $[1,5,17]$. When the closure caused by the bezoar is in the terminal region of the colon, a conservative treatment such as an enema or a resin is first used to remove the bezoar [17]. If this conservative treatment fails, then colonoscopy can be performed for the removal [17]. When complications, such as strangulated intestinal obstruction, massive melena, or peritonitis, occur, along with early surgery, administration of broad-spectrum antibiotics and fluids and electrolyte correction are required $[3,4,8]$.

In our case, by performing abdominal computed tomography on the patient suspected of suffering from acute bowel obstruction, we were able to confirm a calcified foreign mass with a mottled gas pattern, and we easily diagnosed a bezoar-caused bowel obstruction. An enema did not remove the bezoar, and because there were no signs of a strangulated intestinal obstruction or peritonitis, a colonoscopy was performed and successfully removed the bezoar.

Among the causes of bowel obstruction, bezoar-caused obstructions can be difficult to diagnose because they are very rare [1]. When the patient suffers from gastrointestinal obstruction, is elderly, has used medications that can decrease gastrointestinal mobility, has undergone gastrointestinal surgery, or has diabetic gastropathy, muscular atrophy, or cerebral infarction, the possibility of gastrointestinal obstruction being caused by a bezoar needs to be considered, and computed tomography is thought to be useful for diagnosing an obstruction caused by a bezoar and for differentiating it from other obstructions due to other causes.

\section{CONFLICT OF INTEREST}

No potential conflict of interest relevant to this article was reported.

\section{REFERENCES}

1. Bedioui H, Daghfous A, Ayadi M, Noomen R, Chebbi F, Rebai W, et al. A report of 15 cases of small-bowel obstruction secondary to phytobezoars: predisposing factors and diagnostic difficulties. Gastroenterol Clin Biol 2008;32:596-600.

2. Mohammad MA. Rectosigmoid lithobezoar in a eight-year-old. Afr J Paediatr Surg 2010;7:38-9.

3. Kim JP, Park JG, Hong SC, Lee KU, Park JW, Kwon OJ, et al. Clinical analysis of Bezoars. Korean J Gastroenterol 1991;23:32-8.

4. Bala M, Appelbaum L, Almogy G. Unexpected cause of large bowel obstruction: colonic bezoar. Isr Med Assoc J 2008;10:829-30.

5. Kim MC, Chae HS, Tae HJ, Park SH, Jang JW, Lee KM, et al. Small intestinal obstruction due to phytobezoars: case reports. Korean J Gastrointest Endosc 2001;22:182-6.

6. Lee SJ, Chu SJ, Tsai SH. Colonic phytobezoar. BMJ Case Rep 2009; 2009:bcr2006039412.

7. De Bakey M, Ochsner A. Bezoars and concretions: a comprehensive review of the literature with an analysis of 303 collected cases and a presentation of 8 additional cases. Surgery 1938;4:934-63.

8. Erzurumlu K, Malazgirt Z, Bektas A, Dervisoglu A, Polat C, Senyurek $\mathrm{G}$, et al. Gastrointestinal bezoars: a retrospective analysis of 34 cases. World J Gastroenterol 2005;11:1813-7.

9. Ha SS, Lee HS, Jung MK, Jeon SW, Cho CM, Kim SK, et al. Acute intestinal obstruction caused by a persimmon phytobezoar after dissolution therapy with Coca-Cola. Korean J Intern Med 2007;22: 300-3.

10. Lee J. Bezoars and foreign bodies of the stomach. Gastrointest Endosc Clin N Am 1996;6:605-19.

11. Hui MS, Perng HL, Choi WM, Chem LK, Yang KC, Chen TJ. Afferent loop syndrome complicated by a duodenal phytobezoar after Billroth-II subtotal gastrectomy. Am J Gastroenterol 1997;92:1550-2.

12. Minty B, Kelly L. Rectal bezoars in children. CMAJ 2010;182:E532.

13. Kim JH, Ha HK, Sohn MJ, Kim AY, Kim TK, Kim PN, et al. CT findings of phytobezoar associated with small bowel obstruction. Eur Radiol 2003;13:299-304.

14. Billaud Y, Pilleul F, Valette PJ. Mechanical small bowel obstruction due to bezoars: correlation between CT and surgical findings. J Radiol 2002;83:641-6.

15. Verstandig AG, Klin B, Bloom RA, Hadas I, Libson E. Small bowel phytobezoars: detection with radiography. Radiology 1989;172: 705-7.

16. Suri S, Gupta S, Sudhakar PJ, Venkataramu NK, Sood B, Wig JD. Comparative evaluation of plain films, ultrasound and CT in the diagnosis of intestinal obstruction. Acta Radiol 1999;40:422-8.

17. Andrus CH, Ponsky JL. Bezoars: classification, pathophysiology, and treatment. Am J Gastroenterol 1988;83:476-8. 